\title{
OPTIMAL LIQUIDATION OF A CALL SPREAD
}

\author{
ERIK EKSTRÖM, ${ }^{* * *}$ Uppsala University \\ CARL LINDBERG, ${ }^{* * *}$ Chalmers University of Technology \\ JOHAN TYSK, ${ }^{*}$ Uppsala University \\ HENRIK WANNTORP, ${ }^{* * * *}$ Swedbank Markets
}

\begin{abstract}
We study the optimal liquidation strategy for a call spread in the case when an investor, who does not hedge, believes in a volatility that differs from the implied volatility. The liquidation problem is formulated as an optimal stopping problem, which we solve explicitly. We also provide a sensitivity analysis with respect to the model parameters.
\end{abstract}

Keywords: Optimal stopping; call spread; Bachelier model

2010 Mathematics Subject Classification: Primary 91B28

Secondary $60 \mathrm{G} 40$

\section{Introduction}

In recent years, volatility has emerged as an asset class in its own right. As option markets are becoming increasingly liquid, new financial instruments such as variance swaps and volatility futures are introduced. However, exposure to volatility is still typically taken by various option strategies. One of the most common strategies is to take a position in an option spread. A call spread is a long position in a call option and a short position in another call on the same underlying and with the same maturity, but with a different strike price. A put spread is defined analogously. By construction, the spread has both limited profit and limited loss. As a consequence, it is considered a conservative and cautious strategy.

In any complete market model, options that trade at an implied volatility different from the true volatility allow for arbitrage opportunities. In practice, however, there is risk that cannot be hedged away due to incompleteness and market imperfections such as illiquidity and transaction costs. Options are therefore often traded at an implied volatility $\tilde{\sigma}$ that is larger than the true future realized volatility $\sigma$; compare [1] and the references therein. We consider an agent looking to benefit from such a mispricing by investing in an appropriate call spread. The agent is assumed to be a true buyer, i.e. an investor who will not attempt to hedge market positions.

The call spread price is convex as a function of the underlying asset price for small values of the asset and concave for large values. Consequently, the price increases in volatility for small asset values and decreases for large values. Therefore, the market price corresponding to the implied volatility $\tilde{\sigma}$ is lower than the expected payoff corresponding to the volatility $\sigma$ for large asset values, and the investor would hold position. On the other hand, for small asset values,

Received 23 June 2009; revision received 26 December 2009.

* Postal address: Department of Mathematics, Uppsala University, Box 480, SE-751 06 Uppsala, Sweden.

Support from the Swedish Research Council (VR) is gratefully acknowledged.

** Email address: ekstrom@math.uu.se

*** Postal address: Chalmers University of Technology, SE-412 96 Göteborg, Sweden.

**** Postal address: Quantitative Research, Swedbank Markets, SE-105 34 Stockholm, Sweden. 
the market price is high and the investor would want to liquidate his/her position. In this paper we study the corresponding optimal liquidation strategy.

In the next section we set up a mathematical model for the situation described above. In order to arrive at explicit formulae, we make the following assumptions. First, the payoff of the long and the short calls is replaced by the payoff of a digital option. This is a natural simplification of the problem since the payoff of a digital option can be approximated by a long position in a call option with strike $K$, and a short position in a call option with the next quoted strike which is larger than $K$. For equity index options, and liquid single name options, strikes are typically close to each other, so this approximation is reasonably good. Second, we use the Bachelier model for the underlying asset. While the Black-Scholes model is the industry benchmark model for options, it has several drawbacks from a modeling perspective. One such drawback is that the Black-Scholes model has a constant volatility, whereas volatility typically exhibits a negative correlation with the underlying. The Bachelier model incorporates at least some aspects of this important feature, and it is analytically very tractable. A theoretical deficiency of the Bachelier model is that it allows for negative asset prices with positive probability. However, for relatively short times to expiry this objection is of no practical importance; compare the remark in Section 2.

We show that the problem of when to optimally liquidate the call spread can be formulated as an optimal stopping problem with finite time horizon. To solve the optimal stopping problem, we first perform a deterministic space and time change which is closely related to the transformations considered in [3]. The resulting problem has an infinite horizon and involves a time-homogeneous Ornstein-Uhlenbeck process, and is thus explicitly solvable using standard methods from optimal stopping theory. Transforming back to the original coordinates, it is shown that the optimal liquidation boundary is of the square root type; compare Theorem 2.1, below. We also study the dependence of the size of the continuation region on model parameters. Finally, in Section 3 we investigate how the strike $K$ should be chosen.

\section{The optimal liquidation problem}

As explained above, we use the Bachelier model for the underlying asset price $X$, i.e.

$$
\mathrm{d} X_{t}=\sigma \mathrm{d} W_{t}
$$

where $\sigma>0$ is a constant volatility and $W$ denotes a standard Brownian motion. The assumption of zero drift is motivated by short maturities and computational simplicity. Moreover, the call spread considered below is typically used by agents with a certain view on the volatility, whereas an investor with a belief in a significant drift would invest differently.

Remark. A theoretical deficiency of the Bachelier model is that asset price might become negative with positive probability. However, this feature is of minor practical importance since the probability is tiny. For example, the probability that $X_{t}=y+0.2 y W_{t}$ will ever fluctuate down below 0 during one quarter of a year is $2 \Phi(-1 / 0.2 \sqrt{1 / 4})=2 \Phi(-10)$, which is negligible.

Consider a digital option struck at $K$, i.e. an option paying the amount $g\left(X_{T}\right)$ at time $T$, where

$$
g(x)= \begin{cases}1 & \text { if } x \geq K \\ 0 & \text { if } x<K\end{cases}
$$


If the underlying is worth $y$ at time $t<T$ then the expected payoff of the option is

$$
\mathrm{P}\left(y+\sigma W_{T-t} \geq K\right)=\Phi\left(\frac{y-K}{\sigma \sqrt{T-t}}\right),
$$

where

$$
\Phi(x)=\int_{-\infty}^{x} \varphi(z) \mathrm{d} z \text { and } \varphi(x)=\frac{1}{\sqrt{2 \pi}} \mathrm{e}^{-x^{2} / 2}
$$

denote the distribution and density function of a standard normal random variable. We consider a situation with a high implied volatility $\tilde{\sigma}>\sigma$. More precisely, we assume that the market price of the call spread is

$$
\Pi(t, y)=\Phi\left(\frac{y-K}{\tilde{\sigma} \sqrt{T-t}}\right)
$$

if the underlying is worth $y$ at time $t<T$. Note that, for simplicity, we assume a zero risk-free rate.

We consider the case of an investor who wants to make a profit by trading on the belief that the true future realized volatility $\sigma$ is smaller than the implied volatility $\tilde{\sigma}$. We also assume that the investor is a true buyer in the sense that he/she does not hedge their position, but rather holds the call spread until his/her market view makes liquidation optimal. At any instant $t$ the investor may choose to liquidate their position, thereby receiving the market value $\Pi\left(t, X_{t}\right)$ of the digital option. On the other hand, if the investor chooses to hold the option until time $T$, then he/she receives the amount $g\left(X_{T}\right)$. We are thus faced with the optimal stopping problem

$$
\begin{aligned}
V(t, x) & =\sup _{t \leq \tau \leq T} \mathrm{E}_{t, x}\left[\Pi\left(\tau, X_{\tau}\right) \mathbf{1}_{\{\tau<T\}}+g\left(X_{T}\right) \mathbf{1}_{\{\tau=T\}}\right] \\
& =\sup _{t \leq \tau \leq T} \mathrm{E}_{t, x}\left[\Phi\left(\frac{X_{\tau}-K}{\tilde{\sigma} \sqrt{T-\tau}}\right) \mathbf{1}_{\{\tau<T\}}+g\left(X_{T}\right) \mathbf{1}_{\{\tau=T\}}\right],
\end{aligned}
$$

where $\tau$ denotes a stopping time with respect to the filtration generated by $X$ and the indices denote that $X_{t}=x$.

Remark. The optimal stopping problem (2.1) can alternatively be formulated as a regime switching problem. To see this, note that the quantity $\Phi\left(\left(X_{\tau}-K\right) / \tilde{\sigma} \sqrt{T-\tau}\right)$ represents the probability of ending up above $K$ at time $T$ if a Brownian motion with volatility $\tilde{\sigma}$ starts at $X_{\tau}$ at time $\tau$. Thus, problem (2.1) is equivalent to the problem of deciding a moment at which the volatility of a Brownian motion increases from $\sigma$ to $\tilde{\sigma}$, and where the objective is to maximise the probability that the Brownian motion ends up above $K$.

To solve problem (2.1), consider the process $Y$ given by

$$
Y_{u}=\frac{X_{u}-K}{\tilde{\sigma} \sqrt{T-u}}
$$

for $u \in[t, T)$. Note that Itô's formula yields

$$
\mathrm{d} Y_{u}=\frac{Y_{u}}{2(T-u)} \mathrm{d} u+\frac{\sigma}{\tilde{\sigma} \sqrt{T-u}} \mathrm{~d} W_{u},
$$

and that $\lim _{u \rightarrow T} Y_{u}$ is either $+\infty$ or $-\infty$. By performing the deterministic time change $u=\rho_{s}$, where

$$
\rho_{s}-t=(T-t)\left(1-\mathrm{e}^{-s}\right)
$$


we find that the process

$$
Z_{s}=Y_{\rho_{s}}, \quad s \geq 0
$$

satisfies

$$
\mathrm{d} Z_{s}=\frac{1}{2} Z_{s} \mathrm{~d} s+\beta \mathrm{d} W_{s}^{\prime}
$$

for some standard Brownian motion $W^{\prime}$ and $\beta=\sigma / \tilde{\sigma}<1$. The optimal stopping problem (2.1) satisfies

$$
V(t, x)=F\left(\frac{x-K}{\tilde{\sigma} \sqrt{T-t}}\right),
$$

where $F$ is given by

$$
F(z)=\sup _{0 \leq \tau \leq \infty} \mathrm{E}_{z}\left[\Phi\left(Z_{\tau}\right)\right]
$$

Here the supremum is taken over all random times which are stopping times with respect to the filtration generated by $Z$, and we use the convention that $\Phi\left(Z_{\tau}\right)=\lim _{s \rightarrow \infty} \Phi\left(Z_{s}\right)$ on the set $\{\tau=\infty\}$.

An application of Itô's formula shows that

$$
\mathrm{d} \Phi\left(Z_{s}\right)=\frac{1}{2} \varphi\left(Z_{s}\right) Z_{s}\left(1-\beta^{2}\right) \mathrm{d} s+\beta \varphi\left(Z_{s}\right) \mathrm{d} W_{s}^{\prime} .
$$

Since $\beta<1$, the drift has the same sign as $Z_{s}$, and we therefore expect the optimal stopping time in the perpetual optimal stopping problem (2.4) to be of the form

$$
\tau_{B}=\inf \left\{t \geq 0: Z_{t} \leq B\right\}
$$

for some constant boundary $B$. Moreover, general theory for optimal stopping problems (see, for example, [4]) suggests that the value function $F$ and the optimal stopping boundary $B$ should satisfy the free boundary problem

$$
\begin{cases}\beta^{2} F^{\prime \prime}(z)+z F^{\prime}(z)=0 & \text { if } z>B \\ F(z)=\Phi(z) & \text { if } z=B \\ F^{\prime}(z)=\varphi(z) & \text { if } z=B .\end{cases}
$$

The general solution to the ordinary differential equation $\beta^{2} F^{\prime \prime}+z F^{\prime}=0$ is given by

$$
F(z)=C \Phi\left(\frac{z}{\beta}\right)+D,
$$

and since the solution should satisfy $\lim _{z \rightarrow \infty} F(z)=1$, we must have $D=1-C$. Using the conditions at the free boundary $B$, and letting $\Psi(x)=1-\Phi(x)$, we readily verify that the solution to $(2.5)$ is given by $(F, B)$, where

$$
F(z)=1-\frac{\Psi(B)}{\Psi(B / \beta)} \Psi\left(\frac{z}{\beta}\right), \quad z>B,
$$

and $B$ satisfies the equation

$$
G(B)=G\left(\frac{B}{\beta}\right)
$$

where

$$
G(x)=\frac{x \varphi(x)}{\Psi(x)}
$$




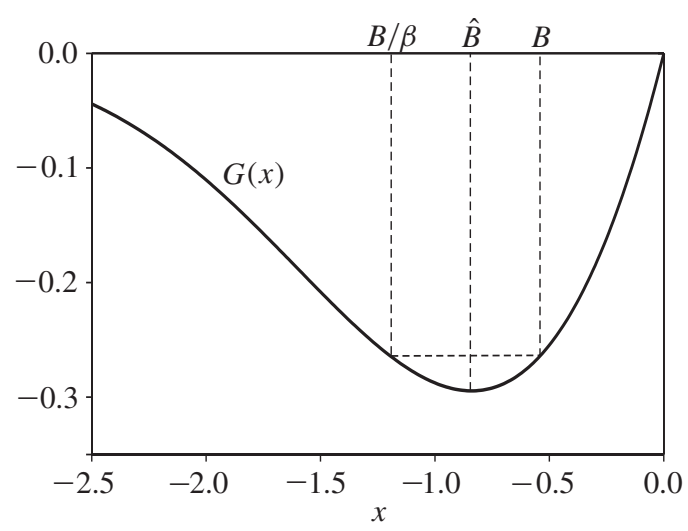

Figure 1: The function $G$.

Remark. Equation (2.6) admits a unique solution, and this solution is negative. Indeed, note that

$$
\frac{\Psi^{2}(x)}{\varphi(x)} G^{\prime}(x)=\left(1-x^{2}\right) \Psi(x)+x \varphi(x)=: h(x) .
$$

It is straightforward to check that $h$ is strictly increasing for $x<0$ and strictly decreasing for $x>0$. Moreover, $h(0)=\frac{1}{2}, h(\infty)=0$, and $h(-\infty)=-\infty$, so $h$ has a unique 0 at some point $\hat{B}<0$. Therefore, $G$ takes its minimal value at $\hat{B}$, and it is strictly increasing for $x>\hat{B}$ and strictly decreasing for $x<\hat{B}$; see Figure 1 . The existence and uniqueness of a solution $B$ to (2.6) now follows.

Below we show that the candidate value function $F$ and the stopping time $\tau_{B}$ determined above indeed solve the optimal stopping problem (2.4). By the time change (2.2) and relation (2.3), we arrive at the solution to the original problem (2.1).

Theorem 2.1. The value function defined in (2.1) is given by

$$
V(t, x)= \begin{cases}1-\frac{\Psi(B)}{\Psi(B / \beta)} \Psi\left(\frac{x-K}{\sigma \sqrt{T-t}}\right), & x>B \tilde{\sigma} \sqrt{T-t}+K, \\ \Phi\left(\frac{x-K}{\tilde{\sigma} \sqrt{T-t}}\right), & x \leq B \tilde{\sigma} \sqrt{T-t}+K,\end{cases}
$$

where $B$ is the unique solution to (2.6). Moreover, the stopping time

$$
\tau_{*}=\inf \left\{t \leq u \leq T: X_{u} \leq B \tilde{\sigma} \sqrt{T-u}+K\right\}
$$

is optimal.

Proof. To verify that the candidate solution

$$
H(z)= \begin{cases}1-\frac{\Psi(B)}{\Psi(B / \beta)} \Psi\left(\frac{z}{\beta}\right) & \text { if } z>B, \\ \Phi(z) & \text { if } z \leq B,\end{cases}
$$

and the optimal stopping time $\tau_{B}$ indeed solve the optimal stopping problem (2.4), note that $H$ belongs to $C^{1}(\mathbb{R}) \cap C^{2}(\mathbb{R} \backslash\{B\})$. Moreover, since the limits $H^{\prime \prime}(B \pm)=\lim _{z \rightarrow B \pm} H^{\prime \prime}(z)$ 
exist and are finite, a generalised Itô formula applies (compare Problem 3.6.24 of [2]) and

$$
\begin{aligned}
H\left(Z_{t}\right) & =H(z)+\beta \int_{0}^{t} H^{\prime}\left(Z_{u}\right) d W_{u}^{\prime}+\frac{1}{2} \int_{0}^{t}\left(\beta^{2} H^{\prime \prime}\left(Z_{u}\right)+Z_{u} H^{\prime}\left(Z_{u}\right)\right) \mathbf{1}_{\left\{Z_{u} \neq B\right\}} \mathrm{d} u \\
& =H(z)+\beta \int_{0}^{t} H^{\prime}\left(Z_{u}\right) \mathrm{d} W_{u}^{\prime}+\frac{1}{2} \int_{0}^{t}\left(1-\beta^{2}\right) Z_{u} \varphi\left(Z_{u}\right) \mathbf{1}_{\left\{Z_{u}<B\right\}} \mathrm{d} u \\
& =H(z)+M_{t}+\Lambda_{t},
\end{aligned}
$$

where $M_{t}$ is a martingale and $\Lambda_{t}$ is a decreasing process (recall that $B$ is negative). Since $H(z) \geq \Phi(z)$ for all $z$, it follows that

$$
\Phi\left(Z_{\tau \wedge n}\right) \leq H\left(Z_{\tau \wedge n}\right) \leq H(z)+M_{\tau \wedge n}
$$

for any stopping time $\tau$ and any nonnegative constant $n$. Therefore, the optional sampling theorem yields

$$
\mathrm{E}_{z}\left[\Phi\left(Z_{\tau \wedge n}\right)\right] \leq H(z)+\mathrm{E}_{z}\left[M_{\tau \wedge n}\right]=H(z) .
$$

An application of the bounded convergence theorem shows that $\mathrm{E}_{z}\left[\Phi\left(Z_{\tau}\right)\right] \leq H(z)$, and, hence, $F(z) \leq H(z)$ for all $z$.

In order to establish the reverse inequality, note that (2.8) yields

$$
\mathrm{E}_{z}\left[H\left(Z_{\tau_{B} \wedge n}\right)\right]=H(z) .
$$

Letting $n \rightarrow \infty$ and using the bounded convergence theorem together with the fact that $H\left(Z_{\tau_{B}}\right)=\Phi\left(Z_{\tau_{B}}\right)$ gives

$$
\mathrm{E}_{z}\left[\Phi\left(Z_{\tau_{B}}\right)\right]=H(z) .
$$

Thus, $H(z)=F(z)$ and $\tau_{B}$ is optimal, as claimed.

Remark. If the investor believes in a larger volatility than does the market, i.e. if $\sigma>\tilde{\sigma}$, then the investor would invest in a bull spread with a strike price $K$ that is larger than the initial asset price. A similar reasoning as above shows that the value function

$$
V(t, x)=\sup _{t \leq \tau \leq T} \mathrm{E}_{t, x}\left[\Phi\left(\frac{X_{\tau}-K}{\tilde{\sigma} \sqrt{T-\tau}}\right) \mathbf{1}_{\{\tau<T\}}+g\left(X_{T}\right) \mathbf{1}_{\{\tau=T\}}\right]
$$

in that case is given by

$$
V(t, x)= \begin{cases}\frac{\Phi(B)}{\Phi(B / \beta)} \Phi\left(\frac{x-K}{\sigma \sqrt{T-t}}\right), & x<B \tilde{\sigma} \sqrt{T-t}+K, \\ \Phi\left(\frac{x-K}{\tilde{\sigma} \sqrt{T-t}}\right), & x \geq B \tilde{\sigma} \sqrt{T-t}+K,\end{cases}
$$

where $\beta=\sigma / \tilde{\sigma}$ and $B$ is the unique solution to $G(-B)=G(-B / \beta)$.

We now study the dependence of $B$ on the parameter $\beta=\sigma / \tilde{\sigma}<1$. The results are illustrated in Figure 2.

Theorem 2.2. The optimal threshold B satisfies the bounds $\hat{B}<B<\beta \hat{B}$, where $\hat{B}$ is the unique value such that $h(\hat{B})=0$. Moreover, $B$ is decreasing in the parameter $\beta=\sigma / \tilde{\sigma}$. Consequently, for a fixed implied volatility $\tilde{\sigma}$, the continuation region is increasing in the volatility $\sigma$. 


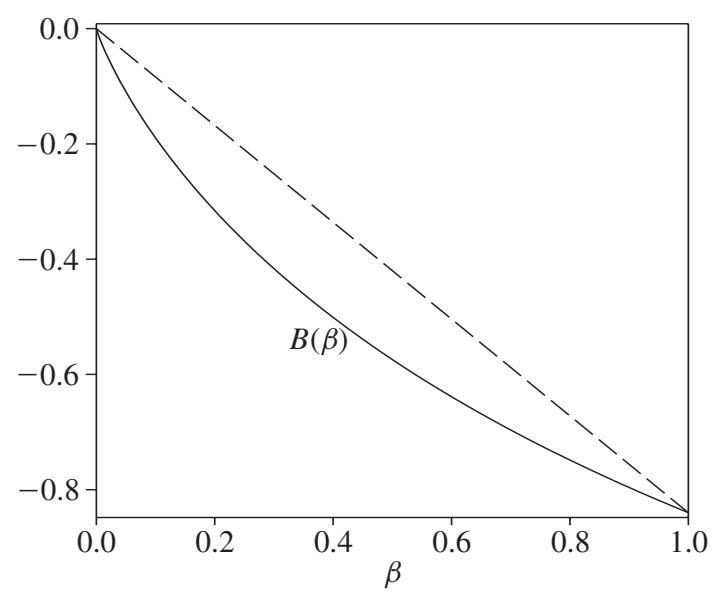

FIGURE 2: The optimal threshold $B$ as a function of $\beta$ and the linear upper bound $\beta \hat{B}$.

Remark. Numerical calculations show that $\hat{B}=-0.8399 \ldots$ One may note that the optimal stopping boundary $B$ tends to $\hat{B}$ as $\beta \rightarrow 1$. However, for $\beta=1$, the problem degenerates since $\Pi\left(t, X_{t}\right)$ is a martingale in that case.

Proof of Theorem 2.2. All statements are straightforward consequences of the fact that $B$ is the unique solution to $G(B)=G(B / \beta)$, where $G$ is as in (2.7).

\section{The optimal choice of the strike price $K$}

In this section we ask the following question: How should the strike price $K$ be chosen in order to maximize the expected profit? To answer this, let $V(0, x ; K)$ be the value function given in Theorem 2.1, and assume that the parameters $x, T, \sigma$, and $\tilde{\sigma}$ are fixed. Let

$$
D(K)=V(0, x ; K)-\Phi\left(\frac{x-K}{\tilde{\sigma} \sqrt{T}}\right)
$$

be the difference between the expected profit from trading the digital option (according to the investor) and the option price (as given by the market).

Theorem 3.1. The function $D(K)$ takes its maximal value for $K^{*}=x+\tilde{\sigma} \sqrt{T} B$.

In Figure 3 a path of a Brownian motion is simulated, and the optimal liquidation time is indicated.

Proof of Theorem 3.1. Differentiating $D$ gives

$$
\begin{aligned}
\tilde{\sigma} \sqrt{T} D^{\prime}(K) & =\varphi\left(\frac{x-K}{\tilde{\sigma} \sqrt{T}}\right)-\frac{\Psi(B)}{\beta \Psi(B / \beta)} \varphi\left(\frac{x-K}{\sigma \sqrt{T}}\right) \\
& =\varphi\left(\frac{x-K}{\tilde{\sigma} \sqrt{T}}\right)-\frac{\varphi(B)}{\varphi(B / \beta)} \varphi\left(\frac{x-K}{\sigma \sqrt{T}}\right) \\
& =\varphi\left(\frac{x-K}{\sigma \sqrt{T}}\right)\left(\varphi\left(\frac{x-K}{\tilde{\sigma} \sqrt{T}}\right)^{1-1 / \beta^{2}}-\varphi(B)^{1-1 / \beta^{2}}\right)(2 \pi)^{(1 / 2)\left(1-1 / \beta^{2}\right)},
\end{aligned}
$$




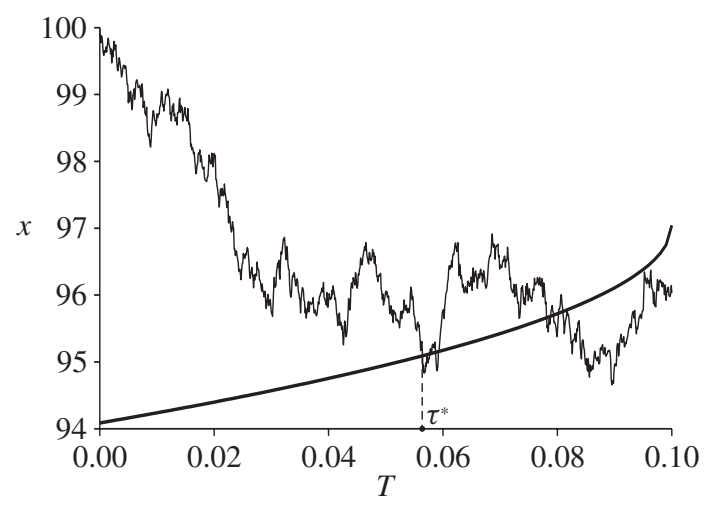

Figure 3: The optimal stopping boundary. Here $x=100, T=0.1, \sigma=10$, and $\tilde{\sigma}=12.5$. Consequently, $\beta=0.8, B=-0.748 \ldots$, and $K^{*}=97.04 \ldots$.

where in the second equality we used (2.6). Consequently, $D^{\prime}(K)=0$ if and only if $K=$ $x+\tilde{\sigma} \sqrt{T} B$ or $K=x-\tilde{\sigma} \sqrt{T} B$. It is straightforward to check that $K=x+\tilde{\sigma} \sqrt{T} B$ gives the global maximum of $D$.

\section{References}

[1] CARr, P. AND Wu, L. (2008). Variance risk premiums. Rev. Financial Studies 22, 1311-1341.

[2] Karatzas, I. And Shreve, S. E. (2000). Brownian Motion and Stochastic Calculus, 2nd edn. Springer, New York.

[3] Pedersen, J. L. And Peskir, G. (2000). Solving non-linear optimal stopping problems by the method of timechange. Stoch. Anal. Appl. 18, 811-835.

[4] Peskir, G. And Shiryaev, A. (2006). Optimal Stopping and Free-Boundary Problems. Birkhäuser, Basel. 